\title{
Identification of 2-Cys Peroxiredoxin (BmTPx-2) as Antioxidant Active Molecule from Babesia microti
}

\author{
Xunan Hai't, Houshuang Zhang ${ }^{1+}$, Zhonghua Wang ${ }^{1}$, Haiyan Gong', Jie Cao', \\ Yongzhi Zhou ${ }^{1}$ and Jinlin Zhou ${ }^{1,2 *}$
}

${ }^{1}$ Key Laboratory of Animal Parasitology of Ministry of Agriculture, Shanghai Veterinary Research Institute, Chinese Academy of Agricultural Sciences, Shanghai, China, ${ }^{2}$ Jiangsu Co-innovation Center for Prevention and Control of Important Animal Infectious Diseases and Zoonoses, Yangzhou, China

OPEN ACCESS

Edited by:

Bang Shen,

Huazhong Agricultural University,

China

Reviewed by:

Xing-Quan Zhu,

Lanzhou Veterinary Research

Institute, Chinese Academy

of Agricultural Sciences, China

Tatsunori Masatani,

Kagoshima University, Japan

*Correspondence:

Jinlin Zhou

jinlinzhou@shvriac.cn

tThese authors have contributed equally to this work.

Specialty section

This article was submitted to Infectious Diseases,

a section of the journal

Frontiers in Microbiology

Received: 27 March 2017 Accepted: 22 September 2017

Published: 10 October 2017

Citation:

Hai X, Zhang H, Wang Z, Gong H,

Cao J, Zhou Y and Zhou J (2017)

Identification of 2-Cys Peroxiredoxin

(BmTPx-2) as Antioxidant Active

Molecule from Babesia microti.

Front. Microbiol. 8:1959.

doi: 10.3389/fmicb.2017.01959
Peroxiredoxins (Prxs) are a family of antioxidant enzymes that reduce peroxides in the presence of thioredoxin, thioredoxin reductase, and nicotinamide adenine dinucleotide phosphate (NADPH) to resist oxidative stress. In this study, we identified and isolated a 2-Cys Prx designated as 'BmTPx-2' from Babesia microti, with a full-length cDNA of $826 \mathrm{bp}$ and an open reading frame of $756 \mathrm{bp}$, which encodes a 251-amino acid protein. BLAST analysis demonstrated that BmTPx-2 shows the typical features of members of the 2-Cys Prx family, which includes harboring two conserved VCP motifs with Cys ${ }^{101}$ and Cys $^{221}$ conserved cysteine residues. Recombinant BmTPx-2 was expressed in Escherichia coli and analyzed by western blot. The antioxidant activity of BmTPx-2 was demonstrated using a mixed-function oxidation system and oxidation of NADPH. Furthermore, BmTPx-2 mRNA expression level in parasites at the erythrocytes and tick stages were analyzed by real-time fluorescence quantitative PCR. Peak BmTPx2 mRNA transcription was detected 8 days after infection at the erythrocyte stage, but not at the tick stage. Taken together, this study characterized BmTPx-2 from B. microti as an antioxidant molecule that was specifically transcribed at the erythrocyte stage.

Keywords: Babesia microti, peroxiredoxin, identification, activity assay, transcription analysis

\section{INTRODUCTION}

Babesia species belong to phylum Apicomplexa, which mainly comprises intra-erythrocytic protozoan parasites (Vannier et al., 2015). Babesia is transmitted by tick bites, blood transfusions, and transplacentally. Babesia was first described in 1888; 5 years later, the tick was confirmed as its transmission vector, and in 1957, human babesiosis was identified (Vannier et al., 2008, 2015). Babesia consists of 100s of species, and among these, Babesia microti is the most frequent cause of human and rodent babesiosis. Human babesiosis has been reported in China (Vannier et al., 2008; Zhou et al., 2013, 2015). In 2012, the entire genome of B. microti was sequenced and mapped (Cornillot et al., 2012, 2013). B. microti causes red blood cell lysis, jaundice, hemolytic anemia, remittent fever, and renal insufficiency, and mortality rate is estimated at $10 \%$ following $B$. microti infection (White et al., 1998; Hatcher et al., 2001; Zhou et al., 2015). Moreover, the fatality rate is higher among those who are immunocompromised or acquire the infection through blood transfusion (Krause et al., 2008; Herwaldt et al., 2011; Leiby, 2011). B. microti has been recognized as an emerging worldwide health threat; therefore, the identification of an efficient and safe method to prevent and treat babesiosis is imperative (Vannier et al., 2008, 2015; Zhou et al., 2013). 
Babesia microti lives in oxygen-rich erythrocytes that generate abundant reactive oxygen species (ROS), which damage biological macromolecules such as nucleic acids and proteins of B. microti, and to counter this effect, the parasite is equipped with anti-oxidation molecules such as thioredoxin, peroxiredoxins (Prxs), glutaredoxins, and superoxide dismutases. Prxs play an important roles against the toxicity of ROS, which are generated during hemoglobin digestion (Storz and Imlay, 1999; Tanaka et al., 2009; Robinson et al., 2010; Masatani et al., 2014, 2016; Wang et al., 2016). Prxs were initially discovered in 1989 in S. typhimurium, with an ability to resolve organic peroxide cumene hydroperoxide. Prxs play an array of functions, and a few have been confirmed in parasites, and the most relevant involves its thioredoxin-dependent peroxidase activity (Rhee and Woo, 2011; Gretes et al., 2012; Angelucci et al., 2016). The reduction of Prx expression by knockout (KO) and RNA interference (RNAi) is lethal to the survival of the parasite, thus confirming that Prxs are crucial to their survival and virulence (Sayed et al., 2006; Castro et al., 2011; Kimura et al., 2013; Hakimi et al., 2014; Usui et al., 2015). Recent studies have demonstrated that the physiological roles of parasite Prxs involve their potential use chemotherapy and as drug targets (Ginsburg et al., 1999; Krauth-Siegel et al., 2005).

Prxs are divided into three types based on the number and position of conserved cysteine residues, namely, 1-Cys, typical 2-Cys and 2-Cys type (Rhee et al., 2001; Wood et al., 2003; Rhee, 2016). The functional unit of typical 2-Cys Prxs is a homodimer (Angelucci et al., 2015, 2016). However, the 2-Cys TPx-2 of B. microti has not been characterized to date. Therefore, the main focus of the present study was to identify and to elucidate the function of the antioxidant biological properties of TPx-2, a novel Prx of B. microti. In this study, we obtained a novel Prx protein from B. microti, which we called 'BmTPx-2.' BLASTp analysis determined that BmTPx2 belongs to the thioredoxin-like superfamily. $B m T P x-2$ is a typical 2-Cys Prx, and amino acid sequence alignment with other 2-Cys Prx indicated that BmTPx-2 also harbored two conserved cysteine residues $\left(\mathrm{Cys}^{101}\right.$ and $\left.\mathrm{Cys}^{221}\right)$, in which Cys $^{101}$ was a peroxidatic acid and $\mathrm{Cys}^{221}$ was a resolving acid.

\section{MATERIALS AND METHODS}

\section{Parasite Culture}

The B. microti strain was obtained from the American Type Culture Collection (ATCC, PRA-99 ${ }^{\mathrm{TM}}$ ) and was injected into Kunming mice. B. microti was maintained in purified mice erythrocytes and the parasite was isolated when the erythrocyte infection rate reached 30-40\%, which was confirmed with Giemsa-stained thin-blood smears.

\section{B. microti cDNA Library Construction and Illumina Sequencing}

Total RNA of B. microti was extracted using TRIzol reagent (Life Technologies Corporation, Carlsbad, CA, United States) and dissolved in RNase-free water. RNA integrity was verified by gel electrophoresis, and quantity was determined using a NanoVue spectrophotometer (GE Healthcare Life Sciences, Pittsburg, PA, United States). cDNA library construction and Illumina sequencing of the samples were performed at Genomics Institute (BGI, Shenzhen, China). RNA sequence libraries were constructed according to Illumina manufacturer's instructions for 150-bp paired-ends and then sequenced using Illumina HiSeq 2000 (Illumina, United States). A total of 3,024 genes were obtained from transcriptome assembly.

\section{Molecular Cloning of BmTPx-2 and Multiple Sequence Alignment Analysis}

Based on the open reading frame (ORF) sequence of BmTPx-2 downloaded from GenBank (XM_012792398.1), we designed a pair of specific primers: BmTPx-2-F: $5^{\prime}-\mathrm{TT}$ CCATGG CA ATG CGG TAC ATA ACA CTT TTA-3', BmTPx-2-R: 5'-TT CTCGAG AGA AGT TTG GCC AAA AGC CTC-3', NcoI and XhoI sites are underlined. We amplified the ORF full sequence using the cDNA library as template. Then, the purified PCR products were ligated onto a pMD-18T vector (TaKaRa, Japan) and sequenced. Multiple sequence alignment of BmTPx-2 was performed with 2-Cys TPx2 of Plasmodium falciparum (Pf: GenBank Accession number BAA97121.1), 2-Cys TPx-2 of Theileria parva (Tp: XP_765704.1), and 2-Cys TPx-2 of Tamarix hispida (Th: FH74407.1), TPx-2 of Toxoplasma gondii (Tg: AG25678.2).

\section{Full-Length Sequence of BmTPx-2}

To obtain the full sequence of the $3^{\prime}$ full sequence and $5^{\prime}$ full sequence of BmTPx-2, the $5^{\prime}$ and $3^{\prime}$ regions of the mRNA were amplified using a SMARTER RACE cDNA amplification kit (Clontech, San Jose, CA, United States), following the manufacturer's instructions. The primers were designed based on the ORF sequence earlier obtained. The primer sequences were as follows: GSP1: 5'-GACAGCCATGTCACCTCCCT-3', GSP2: 5' -CAAGTATGTGCCGTATATTTCCAAA-3', and GSP3: 5'-GCGAGTTTGTAAAGTGAATGTATGG-3' for $5^{\prime}$ rapid amplification of cDNA ends (RACE); GSP1: $5^{\prime}$-TGGGTAGGA GTGTGGATGAGAC-3' and GSP2: 5'-AAGGGTAATAAGGGA ATGGCGGC-3' for $3^{\prime}$ RACE. The resulting PCR products were subsequently purified and ligated onto the pMD-18T vector (TaKaRa, Japan) and sequenced. RACE analyses were performed according to the manufacturer's recommendations for using the $3^{\prime}$ RACE system for rapid amplification of cDNA ends and 5' RACE system for rapid amplification of cDNA Ends (Invitrogen, United States).

\section{Expression and Purification of Recombinant Protein BmTPx-2}

We cloned the ORF of $B m T P x-2$ into the prokaryotic expression vector $\mathrm{pET}-28 \mathrm{a}$. Then, to generate the fusion protein, the recombinant vector $\mathrm{pET}-28 \mathrm{a}-\mathrm{BmTPx}-2$ was transformed into Escherichia coli BL21 cells at $16^{\circ} \mathrm{C}$ by $1 \mathrm{mM}$ isopropylthio- $\beta$-Dgalactoside (IPTG) induction. The fusion protein was purified with NI-NTA agarose beads under native conditions (Merck, United States). 


\section{Antioxidant Activity Assay}

Next, to evaluate the antioxidant activity of the recombinant proteins, a mixed-function oxidation (MFO) assay was performed to determine the ability of $\mathrm{BmTPx}-2$ in protecting DNA from damage in oxidative conditions: the MFO mix reaction buffer contained $8 \mathrm{mM} \mathrm{FeCl}_{3}, 40 \mathrm{mM}$ dithiothreitol (DTT), 20 mM EDTA, 25 mM HEPES (pH 7), 180 ng pBluescript plasmid DNA, and various concentrations of the purification fusion protein $\mathrm{rBmTPx}-2(335,167,83$, and $41 \mu \mathrm{g} / \mathrm{mL})$. After mixing, the reaction buffer was pre-incubated at $37^{\circ} \mathrm{C}$ for $1 \mathrm{~h}, 180 \mathrm{ng}$ pBluescript plasmid DNA was then added, and the mixture was incubated at $37^{\circ} \mathrm{C}$ for another $3 \mathrm{~h}$. Nicking of the supercoiled plasmids was detected by $1 \%$ agarose gel electrophoresis and stained with ethidium bromide.

A standard experiment to identify the antioxidant activity of $\mathrm{rBmTPx}-2$ was performed in a $200-\mu \mathrm{L}$ mixture buffer that contained $375 \mu \mathrm{M}$ of NADPH, $1 \mathrm{mM}$ of $\mathrm{H}_{2} \mathrm{O}_{2}, 6.4 \mu \mathrm{M}$ Trx, $0.14 \mu \mathrm{M}$ TrxR, HEPES-NaOH (pH 7), and $100 \mu \mathrm{g} / \mathrm{mL}$ rBmTPx2 . The negative control group contained $\mathrm{H}_{2} \mathrm{O}$ instead of $\mathrm{rBmTPx}-$ 2 in the assay system. NADPH oxidation was monitored for $30 \mathrm{~min}$ at $30^{\circ} \mathrm{C}$ by measuring the absorbance at a wavelength of $340 \mathrm{~nm}$ using a spectra Max M5 (MD, United States).

\section{Production of Mouse Antiserum against the Recombinant Protein}

Eight-week-old male mice (Slac, China) were used for the production of antiserum against $\mathrm{rBmTPx}-2$. For primary immunization, a mixture of $100 \mu \mathrm{g}$ of $\mathrm{BmTPx}-2$ and an equal volume of Freund's complete adjuvant (Sigma, United States) was intraperitoneally injected into the mice. After 2 weeks, boost immunization was performed in a similar manner as the primary immunization. Serum was collected 20 days after boost immunization. All animal experimental procedures were conducted in accordance with the Guiding Principles of Animal Experimentation in the Shanghai Veterinary Research Institute.

\section{Western Blot Analysis of the Native Protein of Parasites}

Babesia microti-infected RBCs were collected when the infection reached $40-50 \%$. RBCs were pelleted by centrifugation and hemolyzed with a red cell lysis buffer (TianGen, China), shaken repeatedly, and incubated at room temperature for $5 \mathrm{~min}$. After centrifugation, the pellet was washed thrice with PBS until the supernatant was clear, and freeze-thaw cycles in liquid nitrogen and water bath were performed thrice. The RBC lysate was mixed with an equal volume of SDS-PAGE loading buffer and heated to $100^{\circ} \mathrm{C}$ for $10 \mathrm{~min}$, and the protein was separated by SDS-PAGE (15\% gel) and subsequently transferred onto a polyvinylidene difluoride membrane (Immobilon; Millipore). The membranes were blocked with $5 \%$ skim milk diluted in PBS containing $0.05 \%$ Tween (PBST) for $2 \mathrm{~h}$ at $37^{\circ} \mathrm{C}$, and then was washed with $1 \times$ PBST thrice. The membranes were reacted with anti-BmTPx-2 mouse serum at $1: 100$ at $37^{\circ} \mathrm{C}$ for $1 \mathrm{~h}$, followed by goat anti-mouse IgG $(1: 2,000)$. The signals were developed with an Immobilon western chemiluminescence HRP substrate.

\section{Transcription Analysis in the Erythrocyte and Tick Stages by Real-time PCR}

To examine the expression of BmTPx-2 at different stages of infection, 30 6-week-old male mice (Slac, China) were injected with RBCs infected with $B$. microti, and then the mice RBCs were detected by quantitative real-time PCR (qRT-PCR) using the expression of actin mRNA as internal control. We divided 30 mice into 10 groups following B. microti injection, and blood was collected from three mice/group from the 2 nd-11th day after injection. Total RNA of B. microti was extracted using the TRIzol method, and cDNA was synthesized according to the PrimeScript RT reagent kit with gDNA eraser (TaKaRa, Japan). qRT-PCR was performed using cDNA as template and a pair of specific primers, following the recommendations provided in the SYBR premix ExTaq (TaKaRa, Japan) with StepOnePlus PCR system. The experimental values were expressed as relative amounts, and analysis was performed with the $2^{-\Delta \Delta c_{\mathrm{t}}}$ method.

To detect BmTPx-2 gene transcription at the tick stage, five 6-week-old male mice (Slac, China) were injected with RBCs infected with B. microti. Two days after injection, Haemaphysalis longicornis larval ticks were placed on the mice. A total of 54 engorged larval ticks were collected, which were cultivated in a $24^{\circ} \mathrm{C}$ incubator until these exuviated into nymphs. The nymph ticks were placed on the mice, engorged nymph ticks were then collected and cultivated to the adult phase. Next, 24 adult ticks were harvested for DNA extraction with a DNA extraction kit (Shanghai SBS Genetech, China), and the $18 \mathrm{~S}$ RNA of $B$. microti was detected by qRT-PCR. Then, the 30 other adult ticks were divided into 10 groups for RNA extraction, the 10-group RNA for $B m T P x-2$ transcription analysis.

\section{Ethics Statement}

The present work was approved by the Animal Ethics Committee of Shanghai Veterinary Research Institute, Chinese Academy of Agricultural Sciences (CAAS) (No. SHVRI-Mo-0158). The protocol was approved by the Animal Care and Use Committee of the Shanghai Veterinary Research Institute, CAAS.

\section{RESULTS}

\section{Molecular Cloning of BmTPx-2 and Multiple Sequence Alignment Analysis}

The full-length cDNA of $B m T P x-2$ was $826 \mathrm{bp}$, and the ORF length was 756 bp (GenBank Accession Number MF459654), encoding 251 amino acid residues without a signal peptide. The protein had a molecular weight of $28 \mathrm{kDa}$, and a pI of 8.231. BLASTp (NCBI) analysis determined that BmTPx-2 belongs to the 2-Cys Prx2 family. Compared to other known 2-Cys Prxs, we found that BmTPx-2 also contained two conserved Cys VCP motif residues at Cys ${ }^{101}$ and $\mathrm{Cys}^{221}$ (Figure 1). The black boxes with black letters indicate similar residues. The two conserved VCP motifs are underlined. 


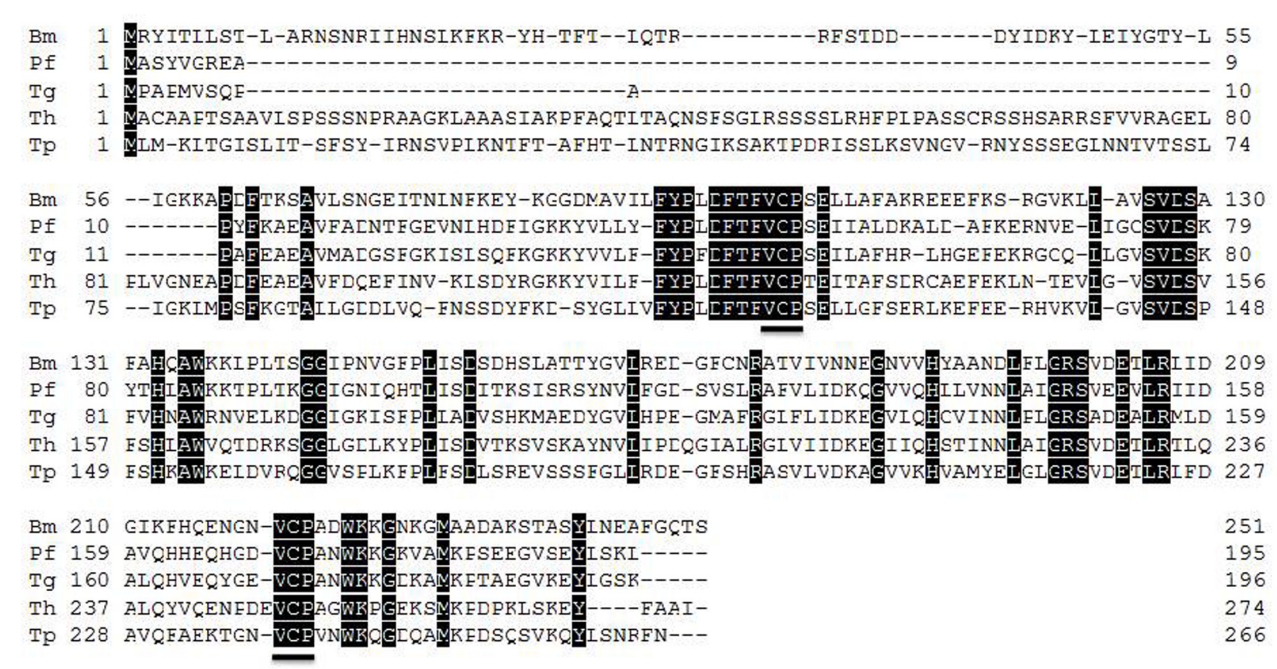

FIGURE 1 | BmTPx-2 multiple sequence alignment analysis. Babesia microti (Bm: MF459654); Plasmodium falciparum (Pf: BAA97121.1); Toxoplasma gondii (Tg: AAG25678.2); Tamarix hispida (Th: AFH74407.1); Theileria parva Muguga (Tp: XP_765704.1). The two conserved VCP motifs are underlined.

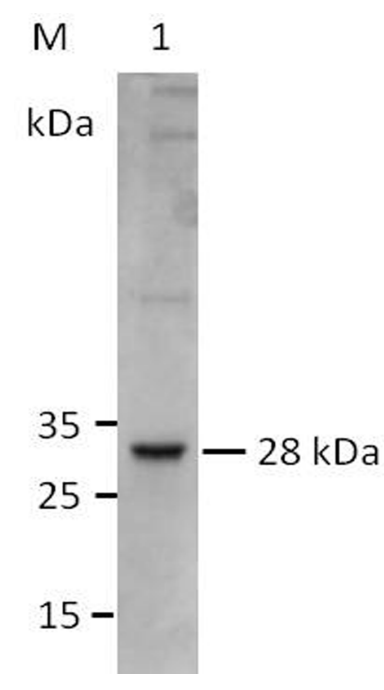

FIGURE 2 | Expression and purification of recombinant BmTPx-2 protein. M, PageRuler prestained protein ladder; 1, rBmTPx-2 was expressed in Escherichia coli BL21 and purified with Ni-NTA agarose beads. Purified rBmTPx-2-His was approximately $28 \mathrm{kDa}$ and was used in subsequent antibody production and antioxidant activity assays.

\section{Expression and Purification of the Recombinant BmTPx-2 Protein}

A fragment of the BmTPx-2 ORF was amplified by PCR, and the product was cloned into a $\mathrm{pET}-28 \mathrm{a}$ vector and transformed into E. coli BL21(DE3). The fusion protein was expressed at $20^{\circ} \mathrm{C}$ for $8 \mathrm{~h}$ and purified using Ni-NTA agarose beads. The fusion and purification of the recombinant proteins was identified by SDSPAGE and standard Coomassie brilliant blue staining. The results indicated that rBmTPx-2 had a molecular mass of about $28 \mathrm{kDa}$ (Figure 2).

\section{Antioxidant Activity of the BmTPx-2 Protein}

Both $\mathrm{FeCl}_{3}$ and DTT of the MFO system produced hydroxyl radicals that can damage the plasmid, and the molecular weight of the nicked plasmid apparently increased (Figure 3A). However, the presence of $\mathrm{rBmTPx}-2$ protected the plasmid from hydroxyl radical-induced damage. When the concentration of $\mathrm{rBmTPx}$ 2 was $335 \mu \mathrm{g} / \mathrm{mL}$, about half of the DNA was undamaged, but when the concentration of BmTPx-2 was reduced to $41 \mu \mathrm{g} / \mathrm{mL}$, nearly all the DNA was damaged. These findings indicated an inverse relationship between the concentration of $\mathrm{rBmTPx}-2$ and the quantity of the nicked plasmid. These results suggest that the antioxidant activity of $\mathrm{rBmTPx}-2$ was concentration-dependent (Figure 3A).

The Trx-TrxR assay system showed a decrease in the absorbance at a wavelength of $340 \mathrm{~nm}$; however, the degree of the decline was not significant, in which after $40 \mathrm{~min}$, the absorbance decreased about 0.32 . The absorbance of the negative control group decreased to about 0.08 compared to that of the BmTPx-2 group (Figure $3 \mathbf{B}$ ).

\section{Western Blot Analysis of the Native BmTPx-2 Protein of Parasites}

Babesia microti native BmTPx-2 protein extracted from RBCs of mice infected with $B$. microti was detected using an antiBmTPx-2 mouse antiserum, whereas the negative control showed no specific bands (Figure 4). The results demonstrated that the molecular weight of BmTPx-2 of B. microti were about 28 or $24 \mathrm{kDa}$.

\section{Transcription Analysis at the Erythrocyte Stages and Tick Stages}

The level of $B m T P x-2$ mRNA expression in erythrocytes was maintained at a relatively stable level from the 2 nd to 7 th day 


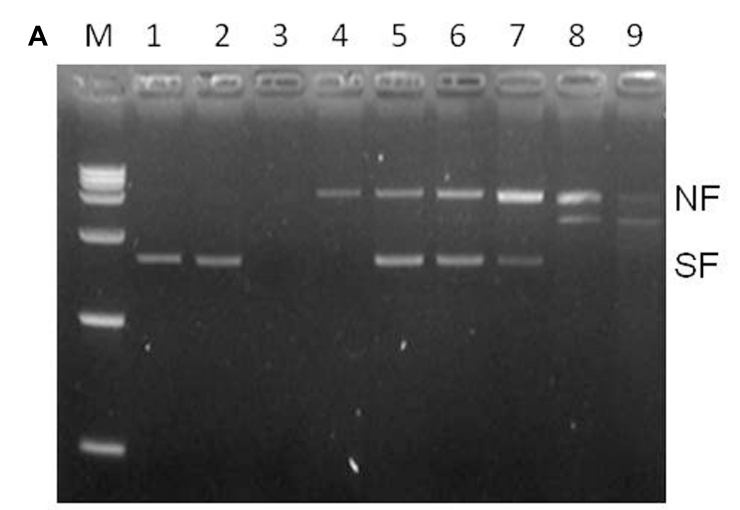

\section{B}

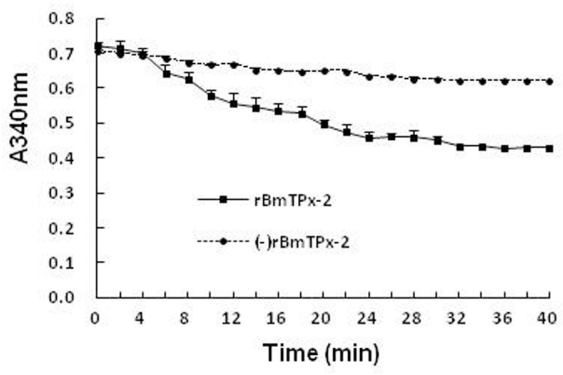

FIGURE 3 | Antioxidant activity of the BmTPx-2 protein. (A) DNA protection experiment analyzed by MFO system. M, DNA marker; (1) DNA+ $\mathrm{H}_{2} \mathrm{O}$; (2) DNA+ DTT; (3) DNA+ FeCl ; (4) DNA+MFO; (5) $335 \mu \mathrm{g} / \mathrm{mL}$ rBmTPx-2+MFO+DNA; (6) $167 \mu \mathrm{g} / \mathrm{mL} \mathrm{rBmTPx-2+MFO+DNA;} \mathrm{(7)} 83 \mu \mathrm{g} / \mathrm{mL}$ rBmTPx-2+MFO+DNA; (8) 55 $\mu \mathrm{g} / \mathrm{mL}$ rBmTPx-2+MFO+DNA; (9) $42 \mu \mathrm{g} / \mathrm{mL}$ rBmTPx-2+MFO+DNA. The nicked form (NF) and supercoiled form (SF) of the plasmids are indicated on the right and the triangle shows the increasing concentration of the recombinant proteins. (B) Antioxidant function of identification by using a Trx-TrxR assay system. The solid line indicates the antioxidant function of rBmTPx-2; the dotted line indicates the absence of rBmTPx-2. NADPH oxidation was monitored as the decrease in (A) 340 nm. The Data are presented as the means of three independent experiments.

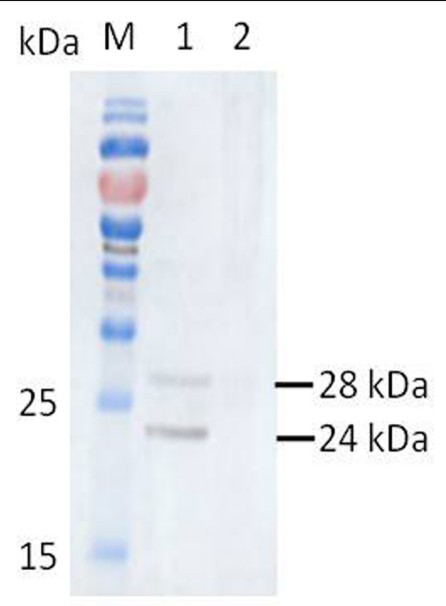

FIGURE 4 | Western blot analysis for native protein of parasites. M, PageRuler prestained protein ladder; (1) RBCs from mice infected with B. microti; (2) RBCs from mice uninfected with $B$. microti.

(Figure 5). BmTPx-2 expression peaked on the 8th day and then decreased on the 9 th day. On the other hand, no B. microti $18 S$ DNA was detected in 24 adult ticks by using RT-PCR, and we also could not detect the BmTPx-2 transcription of B. microti among 10 adult RNA.

\section{DISCUSSION}

Prx plays important roles in protecting parasites from damage induced by ROS in host erythrocytes, as well as in B. microti (Tanaka et al., 2009; Robinson et al., 2010). Prxs were initially discovered in 1989 to have an ability to resolve organic peroxide cumene hydroperoxide. Although not all of the role of Prxs have been confirmed in parasites, its major function involves

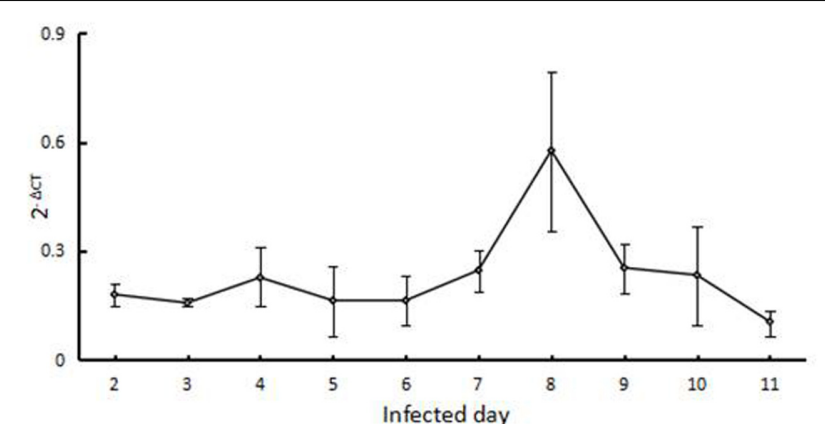

FIGURE 5 | Transcription analysis at the erythrocyte stage. $x$-axis refers to the day of infection of mouse RBCs with $B$. microti; the $y$-axis refers to the relative BmTPx-2 expression level in mouse RBCs at different days after infection with B. microti by using the $2^{-\Delta \Delta C_{\mathrm{T}}}$ mean.

thioredoxin-dependent peroxidase activity (Gretes et al., 2012; Angelucci et al., 2016). In the present study, we obtained the BmTPx-2 full-length cDNA, which comprised a 756-bp long ORF that encodes a 251-amino acid protein without a signal peptide, with a predicted molecular weight of $28 \mathrm{kDa}$. BLASTp analysis revealed that BmTPx-2 belongs to the 2-Cys Prx family, contained two VCP motifs, and the conserved cysteine residues were $\mathrm{Cys}^{101}$ and $\mathrm{Cys}^{221}$, which is similar to that of the other 2-Cys Prxs. In our previous study, we described the identification and functional study of a novel 2-Cys peroxiredoxin (BmTPx-1) of B. microti (Zhang et al., 2016). In this study, the BLASTp results showed that BmTPx-2 had $38 \%$ sequence similarity with BmTPx-1, $48 \%$ with of Peroxiredoxin-2 (Prx2) of Babesia sp. Xinjiang (ORM40258.1), 42\% with Thioredoxin peroxidase 2 (TPx-2) of P. falciparum 3D7 (XP_001350554.1). The BmTPx-2 showed highest homology with Prx2 (TPx-2), so we named this novel gene as BmTPx-2. 
To explore the enzymatic activity of BmTPx-2, we prepared a recombinant BmTPx-2 (rBmTPx-2) protein, and its antioxidant activity was evaluated using an MFO system, which showed that rBmTPx-2 was capable of protecting plasmids from damage, thereby indicative of BmTPx-2 antioxidant activity. BmTPx-2 possibly acts as an antioxidant enzyme that catalyzes hydrogen peroxide in B. microti. The main function of Prxs in parasites involves resolving $\mathrm{H}_{2} \mathrm{O}_{2}$ with Trx, TrxR, and NADPH. Prxs can eliminate hydrogen peroxide with thioredoxin by using thioredoxin as the electron donor (Rhee et al., 2005). Monitoring the oxidation of NADPH suggested that BmTPx-2 plays functions as a peroxide reductase in $B$. microti. Furthermore, western blot analysis revealed that anti-BmTPx-2 mice serum can recognize the native BmTPx-2 protein of $B$. microti. The molecular weight of the native BmTPx-2 protein of B. microti were about 24 or $28 \mathrm{kDa}$. This discrepancy may be due to the different modification used in B. microti. Moreover, the appearance of an extra band in Lane 1 of Figure 4 may be due to the antiserum comprising polyclonal antibodies; therefore, the antiserum may recognize other proteins of $B$. microti besides BmTPx-2. BmTPx-2 transcription during the erythrocyte stage increased from the 2nd to the 7th day and then peaked on the 8th day, thereby suggesting maximal antioxidant response before weakening from the 9th to the 11th day. We could not detect the 18S DNA and BmTPx-2 RNA transcription at the tick stages. This may be due to two reasons: (i) the number of $B$. microti that was ingested by the ticks might have extremely low in that the resulting 18S DNA and BmTPx-2 RNA levels were below the detection limit of the assay; (ii) it is possible that upon placing the ticks on healthy mice, the B. microti pathogens might have been excluded from the salivary glands of ticks. Future studies are necessary to confirm these hypotheses.

\section{REFERENCES}

Angelucci, F., Bellelli, A., Ardini, M., Ippoliti, R., Saccoccia, F., and Morea, V. (2015). One ring (or two) to hold them all - on the structure and function of protein nanotubes. FEBS J. 282, 2827-2845. doi: 10.1111/febs.13336

Angelucci, F., Miele, A. E., Ardini, M., Boumis, G., Saccoccia, F., and Bellelli, A. (2016). Typical 2-Cys peroxiredoxins in human parasites: several physiological roles for a potential chemotherapy target. Mol. Biochem. Parasitol. 206, 2-12. doi: 10.1016/j.molbiopara.2016.03.005

Castro, H., Teixeira, F., Romao, S., Santos, M., Cruz, T., Florido, M., et al. (2011). Leishmania mitochondrial peroxiredoxin plays a crucial peroxidase-unrelated role during infection: insight into its novel chaperone activity. PLOS Pathog. 7:e1002325. doi: 10.1371/journal.ppat.1002325

Cornillot, E., Dassouli, A., Garg, A., Pachikara, N., Randazzo, S., Depoix, D., et al. (2013). Whole genome mapping and re-organization of the nuclear and mitochondrial genomes of Babesia microti isolates. PLOS ONE 8:e72657. doi: 10.1371/journal.pone.0072657

Cornillot, E., Hadj-Kaddour, K., Dassouli, A., Noel, B., Ranwez, V., Vacherie, B., et al. (2012). Sequencing of the smallest Apicomplexan genome from the human pathogen Babesia microti. Nucleic Acids Res. 40, 9102-9114. doi: 10.1093/nar/ gks700

Ginsburg, H., Ward, S. A., and Bray, P. G. (1999). An integrated model of chloroquine action. Parasitol. Today 15, 357-360. doi: 10.1016/S0169-4758(99) 01502-1

Gretes, M. C., Poole, L. B., and Karplus, P. A. (2012). Peroxiredoxins in parasites. Antioxid. Redox. Signal. 17, 608-633. doi: 10.1089/ars.2011.4404
Recent studies knockout and RNAi experiments have determined that Prxs are crucial to the survival and virulence of parasites. Kimura et al. (2013) found that the disruption of the Prxl gene of $P$. falciparum induced hypersensitivity to heat stress. Usui et al. (2015) demonstrated that TPx-2-KO led to death of Plasmodium berghei at the liver stage. To the best of our knowledge, this is the first report that provides evidence that TPx-2 has an antioxidant function against the toxicity of ROS. However, BmTPx-2 is just one of the several Prxs in B. microti, and we believe that a complex relationship among these Prxs may exist, which will be the focus of our future research. Additionally, antioxidant Prxs play other functions such as molecular chaperones, sensors of peroxides, and modulators of inflammatory response (Angelucci et al., 2016). Therefore, in future studies, we will investigate whether BmTPx-2 is involved in these additional functions in B. microti. In conclusion, this study provides foundational basis of the role of BmTPx-2 in B. microti and may be a potential target in treating babesiosis infections.

\section{AUTHOR CONTRIBUTIONS}

$\mathrm{XH}$ and $\mathrm{HZ}$ conducted gene identification and functional studies. $\mathrm{XH}$ wrote the draft of the manuscript. JZ and HZ corrected the manuscript. ZW, HG, JC, and YZ analyzed the results. All authors have read and approved the final manuscript.

\section{FUNDING}

The National Key Basic Research Program (973 Program) of China (Grant No. 2015CB15030).

Hakimi, H., Suganuma, K., Usui, M., Masuda-Suganuma, H., Angeles, J. M., Asada, M., et al. (2014). Plasmodium knowlesi thioredoxin peroxidase 1 binds to nucleic acids and has RNA chaperone activity. Parasitol. Res. 113, 3957-3962. doi: 10.1007/s00436-014-4060-0

Hatcher, J. C., Greenberg, P. D., Antique, J., and Jimenez-Lucho, V. E. (2001). Severe babesiosis in Long Island: review of 34 cases and their complications. Clin. Infect. Dis. 32, 1117-1125. doi: 10.1086/ 319742

Herwaldt, B. L., Linden, J. V., Bosserman, E., Young, C., Olkowska, D., and Wilson, M. (2011). Transfusion-associated babesiosis in the United States: a description of cases. Ann. Intern. Med. 155, 509-519. doi: 10.7326/0003-4819155-8-201110180-00362

Kimura, R., Komaki-Yasuda, K., Kawazu, S., and Kano, S. (2013). 2-Cys peroxiredoxin of Plasmodium falciparum is involved in resistance to heat stress of the parasite. Parasitol. Int. 62, 137-143. doi: 10.1016/j.parint.2012. 11.005

Krause, P. J., Gewurz, B. E., Hill, D., Marty, F. M., Vannier, E., Foppa, I. M., et al. (2008). Persistent and relapsing babesiosis in immunocompromised patients. Clin. Infect. Dis. 46, 370-376. doi: 10.1086/525852

Krauth-Siegel, R. L., Bauer, H., and Schirmer, R. H. (2005). Dithiol proteins as guardians of the intracellular redox milieu in parasites: old and new drug targets in trypanosomes and malaria-causing plasmodia. Angew Chem. Int. Ed. Engl. 44, 690-715. doi: 10.1002/anie.200300639

Leiby, D. A. (2011). Transfusion-transmitted Babesia spp.: bull's-eye on Babesia microti. Clin. Microbiol. Rev. 24, 14-28. doi: 10.1128/CMR.00 022-10 
Masatani, T., Asada, M., Hakimi, H., Hayashi, K., Yamagishi, J., Kawazu, S., et al. (2016). Identification and functional analysis of a novel mitochondrialocalized 2-Cys peroxiredoxin, BbTPx-2, from Babesia bovis. Parasitol. Res. 115 , 3139-3145. doi: 10.1007/s00436-016-5071-9

Masatani, T., Asada, M., Ichikawa-Seki, M., Usui, M., Terkawi, M. A., Hayashi, K., et al. (2014). Cloning and characterization of a 2-Cys peroxiredoxin from Babesia gibsoni. J. Vet. Med. Sci. 76, 139-143. doi: 10.1292/jvms.13-0274

Rhee, S. G. (2016). Overview on Peroxiredoxin. Mol. Cells. 39, 1-5. doi: 10.14348/ molcells.2016.2368

Rhee, S. G., Chae, H. Z., and Kim, K. (2005). Peroxiredoxins: a historical overview and speculative preview of novel mechanisms and emerging concepts in cell signaling. Free Radic. Biol. Med. 38, 1543-1552. doi: 10.1016/j.freeradbiomed. 2005.02.026

Rhee, S. G., Kang, S. W., Chang, T. S., Jeong, W., and Kim, K. (2001). Peroxiredoxin, a novel family of peroxidases. IUBMB Life 52, 35-41. doi: 10. 1080/15216540252774748

Rhee, S. G., and Woo, H. A. (2011). Multiple functions of peroxiredoxins: peroxidases, sensors and regulators of the intracellular messenger $\mathrm{H}_{2} \mathrm{O}_{2}$, and protein chaperones. Antioxid. Redox. Signal. 15, 781-794. doi: 10.1089/ars.20 10.3393

Robinson, M. W., Hutchinson, A. T., Dalton, J. P., and Donnelly, S. (2010). Peroxiredoxin: a central player in immune modulation. Parasite Immunol. 32, 305-313. doi: 10.1111/j.1365-3024.2010.01201.x

Sayed, A. A., Cook, S. K., and Williams, D. L. (2006). Redox balance mechanisms in Schistosoma mansoni rely on peroxiredoxins and albumin and implicate peroxiredoxins as novel drug targets. J. Biol. Chem. 281, 17001-17010. doi: $10.1074 /$ jbc.M512601200

Storz, G., and Imlay, J. A. (1999). Oxidative stress. Curr. Opin. Microbiol. 2, 188-194. doi: 10.1016/S1369-5274(99)80033-2

Tanaka, M., Sakurai, T., Yokoyama, N., Inoue, N., and Kawazu, S. (2009). Cloning and characterization of peroxiredoxin in Babesia bovis. Parasitol. Res. 105, 1473-1477. doi: 10.1007/s00436-009-1587-6

Usui, M., Masuda-Suganuma, H., Fukumoto, S., Angeles, J. M., Hakimi, H., Inoue, N., et al. (2015). Effect of thioredoxin peroxidase-1 gene disruption on the liver stages of the rodent malaria parasite Plasmodium berghei. Parasitol. Int. 64, 290-294. doi: 10.1016/j.parint.2014.09.013
Vannier, E., Gewurz, B. E., and Krause, P. J. (2008). Human babesiosis. Infect. Dis. Clin. North Am. 22, 469-488, viii-ix. doi: 10.1016/j.idc.2008.03.010

Vannier, E. G., Diuk-Wasser, M. A., Ben Mamoun, C., and Krause, P. J. (2015). Babesiosis. Infect. Dis. Clin. North Am. 29, 357-370. doi: 10.1016/j.idc.2015. 02.008

Wang, Q., Zhou, Y., Chen, K., and Ju, X. (2016). Identification and characterization of an atypical 2-cys peroxiredoxin from the silkworm, Bombyx mori. Insect Mol. Biol. 25, 347-354. doi: 10.1111/imb.12224

White, D. J., Talarico, J., Chang, H. G., Birkhead, G. S., Heimberger, T., and Morse, D. L. (1998). Human babesiosis in New York State: review of 139 hospitalized cases and analysis of prognostic factors. Arch. Intern. Med. 158, 2149-2154. doi: 10.1001/archinte.158.19.2149

Wood, Z. A., Schroder, E., Robin Harris, J., and Poole, L. B. (2003). Structure, mechanism and regulation of peroxiredoxins. Trends Biochem. Sci. 28, 32-40. doi: 10.1016/S0968-0004(02)00003-8

Zhang, H., Wang, Z., Gong, H., Cao, J., Zhou, Y., and Zhou, J. (2016). Identification and functional study of a novel 2-cys peroxiredoxin (BmTPx-1) of Babesia microti. Exp. Parasitol. 170, 21-27. doi: 10.1016/j.exppara.2016.08.005

Zhou, X., Li, S. G., Chen, S. B., Wang, J. Z., Xu, B., Zhou, H. J., et al. (2013). Co-infections with Babesia microti and Plasmodium parasites along the ChinaMyanmar border. Infect. Dis. Poverty 2:24. doi: 10.1186/2049-9957-2-24

Zhou, X., Xia, S., Yin, S. Q., and Zhou, X. N. (2015). Emergence of babesiosis in China-Myanmar border areas. Parasit. Vectors 8:390. doi: 10.1186/s13071-0150978-z

Conflict of Interest Statement: The authors declare that the research was conducted in the absence of any commercial or financial relationships that could be construed as a potential conflict of interest.

Copyright (C) 2017 Hai, Zhang, Wang, Gong, Cao, Zhou and Zhou. This is an openaccess article distributed under the terms of the Creative Commons Attribution License (CC BY). The use, distribution or reproduction in other forums is permitted, provided the original author(s) or licensor are credited and that the original publication in this journal is cited, in accordance with accepted academic practice. No use, distribution or reproduction is permitted which does not comply with these terms. 